Conclusion YMSM receiving care at the BCHD STD Clinics are likely to be aware and interested in PrEP but fewer than $10 \%$ may be taking PrEP. Given that STD Clinics serve primarily minority young males who are disproportionately affected by HIV, future work should seek to improve youthfriendly services at public health clinics and leverage awareness and interest in PrEP in order to improve uptake in this vulnerable population.

\section{P5.30 ACCEPTABILITY OF PRE-EXPOSURE PROPHYLAXIS IN GAY MEN AND OTHER MSM IN GUATEMALA CITY}

Ricardo Mendizabal-Burastero, Jose Manuel Aguilar. Colectivo Amigos Contra El Sida, Guatemala

\subsection{6/sextrans-2017-053264.646}

Introduction Pre-exposure prophylaxis (PrEP) is one the most promising HIV interventions, with more than $90 \%$ efficacy with consistent adherence. In Guatemala, after more than 10 years of highly financed interventions, MSM continues to be one of the most affected populations for HIV epidemic. Other interventions, mostly behavioural, haven't changed the course of the HIV epidemic in these population. Our objective was to determinate the acceptability of PrEP in gay men and other MSM in Guatemala city.

Methods Exploratory qualitative study was performed between October and December 2015. 15 MSM were interviewed. Semi-structurated interview of 23 questions regarding: sociodemographic, risk scale, sexual practices and PrEP knowledge. Barriers and facilitators for PrEP were also asked. All participants signed an informed consent. Data analysis was perfomed on Atlas.Ti.

Results Median of age 32 years old, most of the participants reported higher education. Based on CDC guidelines for PrEP, 10 of the participants should be offered PrEP based on their current risks. Condom use was high, and for 3 participants that will be the preferred option always. 13 of them were likely to start PrEP if available, with fears about side effects and cost of the medications. Facilitators for PrEP consumtion was low cost, accessibility of the delivery point, counselling and knowledge about PrEP. To improve adherence, participants recommended phone alarms, text messages, extra pills, flexible or accessible hours for the service.

Conclusion PrEP has a high acceptability among MSM population in Guatemala city. Facilitators and barriers mentions will help to design a successful demonstration project. Based on the reported risks and HIV prevalence in this population, PrEP is an effective needed intervention in these country that can help to change the course of the epidemic. Based on these results, we have started a small fee-based demonstration project since 2016 .

\section{P5.31 SAFETY AND ACCEPTABILITY OF CELLULOSE SULFATEAS A VAGINAL MICROBICIDE IN HIV-INFECTED WOMEN}

Ronald Nyanzi Walakira. Mulago-Mbarara Teaching Hospital, Uganda

\subsection{6/sextrans-2017-053264.647}

Introduction Few studies of topical microbicides have assessed their safety in HIV-infected women. We conducted this study to evaluate the safety and acceptability of $6 \%$ cellulose sulfate
(CS) gel as a vaginal microbicide in sexually abstinent and active HIV-infected women.

Methods Fifty-nine HIV-infected women were enrolled in a randomised double-blind placebo-controlled study comparing $6 \%$ CS to placebo gel used for 14 days. Sexually abstinent women applied gel once or twice daily and sexually active women used gel once daily.

Results CS gel was safe with no reported severe or life-threatening adverse events (AF). Thirty-nine (66\%) of the participants experienced urogenital $\mathrm{AE}$ judged as probably or possibly related to gel. The majority $(51 \%)$ of these participants reported only mild events. Fewer women (62\%) who used CS experienced urogenital AE than those assigned to placebo gel $(70 \%) \quad(p=0.59)$. Eleven $(19 \%)$ women experienced intermenstrual bleeding judged to be probably or possibly related to gel use (four in the CS and seven in the placebo gel group). There was no increase in $\mathrm{AE}$ by frequency of gel use - sexual activity with the exception of abdominal/pelvic pain which was noted more frequently with twice daily use among sexually abstinent women. Women and men found the gel highly acceptable.

Conclusion This Phase I study demonstrated that CS vaginal gel was safe, well tolerated and acceptable by HIV-infected women and their male partners. Thus, further development of CS is warranted as a potential method to prevent HIV transmission and acquisition.

\section{P5.32 BACTERIAL VAGINOSIS AS A RISK FACTOR FOR ACQUIRING SEXUALLY TRANSMITTED DISEASES}

Ssempala Brian Adriane. Centre For Hiv,Std And Tb Prevention, Masaka, Uganda

\subsection{6/sextrans-2017-053264.648}

Introduction Few studies have demonstrated that Bacterial vaginosis (BV) is associated with sexual behaviour risk factors similar to those for other sexually transmitted diseases. In the present study, the prevalence of these in a multivariate analysis of data from sexually active women infected with BV and either Chlamydia trachomatis (CT), Treponema pallidum (syphilis), Neisseria gonorrhoeae (NG) or HIV was observed; NonBV infected women were used as control subjects.

Methods Data from 788 women screened in the SAVVY HIV gel phase III clinica! trial in Accra (West Legon Study Site) from 2012 to 2015 were analysed, Participants were evaluated for the presence of BV, CT, Treponema pallidum (PT), NG, Trichomonas vaginalis (TV) and Human Immunodeficiency Virus (HIV), and interviewed in detail with respect to sexual behaviours after consent forms were signed.

Results This study has shown a high association between BV and HIV $(p<0.01)$ with risk factor $(0.4)$, which does not occurred in the other sexually transmitted diseases like NG, syphilis and Chlamydia with insignificant association $(\mathrm{p}<1)$ and risk factors $(0.6,0.7,0.9)$ respectively. HIV was found to be the most prevalent sexually transmitted disease with $11.2 \%$, Chlamydia 9.2\%, TV $2.3 \%$, Syphilis $1.7 \%$ and NG the least with $1.5 \%$.

Conclusion Bacteria associated with bacterial vaginosis increase female genital-tract infection of HIV but the mechanism by which this happens is not clear. Bacterial vaginosis is not a sexually transmitted disease but predisposes one to HIV infection. It is strongly suggested that all cases of BV both symptomatic and asymptomatic that are presented in the sexual- 